\title{
La utilización de puntajes dínicos para decidir el tratamiento antimiarobiano de la faringitis aguda favorece el ativio sintomático y reduce el uso de antibióticos
}

The use of clinical scores to decide antimicrobial treatments of acute sore throat improves reported symptoms and reduces antibiotic use

Little P, y col. BMJ 2013;347:f5806.

\section{Objetivos}

Determinar la efectividad del uso de puntajes clínicos, asociados o no al uso de pruebas rápidas de detección de antígeno estreptocócico, vs la prescripción extemporánea de antibióticos, en el alivio sintomático y prescripción de antibióticos en pacientes que consultan por faringitis aguda.

\section{Diseño, lugar y pacientes}

Ensayo clínico controlado, multicéntrico, pragmático y abierto, realizado en 48 centros del Reino Unido. Se incluyeron pacientes de tres o más años de edad que consultaron por odinofagia y presentaron eritema faríngeo y/o exudados amigdalinos. Al momento de la consulta los pacientes se evaluaron mediante: presencia y severidad de síntomas (dolor y/o dificultad para tragar, historial de fiebre, catarro de vías aéreas, malestar general, náuseas, vómitos, cefalea, algias corporales, dolor abdominal, trastornos del sueño, interferencia de las actividades diarias) utilizando una escala Likert de cuatro puntos (ningún problema, problema leve, moderado o severo), y presencia de signos (exudados amigdalinos, adenomegalias, adenomegalias dolorosas, fiebre).

\section{Intervención}

Mediante una aleatorización computarizada se asignaron a los pacientes a tres estrategias de abordaje: a) prescripción extemporánea de antibióticos (que se utilizó como comparador); b) utilización de un puntaje clínico para identificar infección estreptocócica; c) utilización de una prueba de antígeno rápido (PAR) guiado por puntaje clínico. La primera estrategia consistía en dejar en la secretaría del centro (una vez finalizada la evaluación) una prescripción de antibióticos con la instrucción de ser retirada a partir del tercer a quinto día de no mejoría o empeoramiento de los síntomas. La segunda estrategia implicaba la utilización de un puntaje clínico, denominado
FeverPAIN, elaborado en estudios previos. El mismo se construyó a partir de los datos obtenidos de la anamnesis y examen físico y otorgaba un rango posible de puntuación de 0 a 5 (ver cuadro 1). Según puntaje de 0 a 1,2 a 3 , o 4 a 5 se recomendaba la no prescripción, prescripción extemporánea, o prescripción inmediata de antibióticos, respectivamente. Para la tercera estrategia se recomendaba según puntajes clínicos de 0 a 1, 2, o $\geq 3$, la no solicitud de PAR y no prescripción de antibióticos, la prescripción extemporánea de antibióticos, y la utilización de PAR para guiar el tratamiento antibiótico solo a aquellos pacientes con resultado positivo, respectivamente. Se instruyó a los pacientes a llevar un diario de síntomas (dolor y/o dificultad para tragar, malestar general, fiebre, trastornos del sueño; registrados con una escala de Likert de 0 a 6) hasta la recuperación o hasta cumplirse 14 días posteriores a la consulta.

Cuadro 1: elementos del puntaje FeverPAIN

\begin{tabular}{c|c} 
Sintoma/signo & Puntaje \\
\hline Ausencia de tos & +1 \\
\hline Fiebre durante las últimas 24 hs & +1 \\
\hline Comienzo de los síntomas 0 a 3 días & +1 \\
\hline Inflamación amigdalina severa & +1 \\
\hline Exudado amigdalino & +1
\end{tabular}

Medición de resultados principales

El resultado primario fue la reducción de la media de máxima odinofagia/dificultad para tragar durante los días dos a cuatro. Como resultados secundarios se evaluaron la duración de los síntomas, utilización de antibióticos, efectos adversos, creencias de los pacientes.

Resultados principales

Se resumen en la tabla 1

Tabla 1: Efecto de tres estrategias clínicas en la evolución sintomática, uso de antibióticos y patrón de consultas de los pacientes mayores de tres años con faringitis aguda.

\begin{tabular}{|c|c|c|c|}
\hline & $\begin{array}{c}\text { Prescripción extemporánea de } \\
\text { antibióticos }\end{array}$ & $\begin{array}{l}\text { Puntaje clinico } \\
\text { (FeverPAIN) }\end{array}$ & $\begin{array}{l}\text { Puntaje clínico + prueba de antigeno } \\
\text { rápido }\end{array}$ \\
\hline \multicolumn{4}{|c|}{ Media de la severidad del dolor y dificultad para tragar entre los días 2 a 4 (escala Likert 0 [sin molestia] a 6 [máxima mulestia]) } \\
\hline Media (DE) & $3,11(1,49)$ & $2,88(1,52)$ & $2,83(1,62)$ \\
\hline Media ajustada* (IC95\%; p) & - & $-0,33(-0,64 a-0,02 ; P=0,04)$ & $-0,30(-0,61$ a 0,$004 ; P=0,05)$ \\
\hline \multicolumn{4}{|c|}{ Duración de los síntomas (en días) puntuados como moderados o severos } \\
\hline Mediana (RIC) & $5(3$ a 7$)$ & $4(2$ a 6$)$ & $4(2$ a 7$)$ \\
\hline Hazard ratio* $(I C 95 \% ; p)$ & 1,00 & $1,30(1,03$ a 1,$63 ; 0,03)$ & $1,11(0,88$ a 1,$40 ; 0,37)$ \\
\hline \multicolumn{4}{|l|}{ Utilización de antibióticos } \\
\hline Porcentaje & $75 / 164(46 \%)$ & $60 / 161(37 \%)$ & $58 / 164(35 \%)$ \\
\hline $\mathrm{RR}^{*}(\mathrm{IC} 95 \% ; \mathrm{p})$ & 1,00 & $0,71(0,50$ a 0,$95 ; 0,02)$ & $0,73(0,52$ a 0,$98 ; 0,03)$ \\
\hline \multicolumn{4}{|c|}{ Creencia en la necesidad de consultar al médico en un episodio futuro (poco probable o menor) } \\
\hline Porcentaje & $62 / 163(38 \%)$ & $54 / 155(35 \%)$ & $64 / 161(40 \%)$ \\
\hline $\mathrm{RR}^{*}$ & - & $0,97(0,71$ a 1,$27 ; 0,85)$ & $1,03(0,76$ a 1,$32 ; 0,86)$ \\
\hline \multicolumn{4}{|c|}{ Reconsulta dentro del mes por dolor de garganta } \\
\hline Porcentaje & $17 / 207(8 \%)$ & $17 / 210(8 \%)$ & $13 / 212(6 \%)$ \\
\hline $\mathrm{RR}^{*} \dagger$ & 1,00 & $0,91(0,47$ a 1,$72 ; 0,78)$ & $0,74(0,36$ a 1,$47 ; 0,40)$ \\
\hline \multicolumn{4}{|c|}{ Reconsulta luego del mes por dolor de garganta (media de seguimiento 0,73 años) } \\
\hline Porcentaje & $31 / 207(15 \%)$ & $26 / 210(12 \%)$ & $34 / 211(16 \%)$ \\
\hline$R^{*} \ddagger$ & 1,00 & $0,79$ (0,47 a 1,$29 ; 0,35)$ & $1,06(0,66$ a 1,$63 ; 0,81)$ \\
\hline
\end{tabular}

RIC: rango intercuartilo. DE: desvío estandar. * Ajustados por la severidad al inicio de los síntomas (dolor de garganta y dificultad para tragar) y fiebre durante las 24 hs previas. † Ajustado además por uso previo de antibióticos. ‡Ajustado por consulta previa por dolor de garganta y duración del seguimiento. 


\section{Conclusiones}

La utilización de puntajes clínicos para decidir el tratamiento antimicrobiano de la faringitis aguda favorece el alivio sintomático y reduce el uso de antibióticos. El agregado de PAR provee beneficios similares, aunque no una ventaja clara sobre el puntaje clínico.
Fuente de financiamiento: National Institute for Health Research Heath Technology Assessment Programme.

Conflicto de interés de los autores: los mismos declaran no existir conflictos potenciales.

\section{Comentario}

Los resultados de este estudio resultan tranquilizadores ya que muestran la efectividad en cuanto a alivio sintomático y reducción del uso de antibióticos de una práctica clínica habitual (el tratamiento antibiótico guiado por puntaje clínico). Esta representa una estrategia posible entre otras (no tratamiento antibiótico; tratamiento antibiótico extemporáneo, PAR y/o cultivo seguido de tratamiento antibiótico en los casos positivos) para las cuales no existe una recomendación uniforme ${ }^{1}$.

Sin embargo es importante tener en cuenta que las estrategias aquí adoptadas implican la posibilidad de no confirmar la presencia de infección estreptocócica en niños o adolescentes mediante cultivo; por ejemplo, un niño con odinofagia y eritema faríngeo, sin tos, sin fiebre, con comienzo de síntomas $>3$ días y $\sin$ exudado amigdalino (puntaje FeverPAIN $=1$ ) no recibiría antibióticos ni sería candidato a la realización de PAR. Esta práctica implementada en algunos países de Europa, como en el caso del Reino Unido², se fundamenta en una muy baja prevalencia de complicaciones no supurativas (fundamentalmente fiebre reumática) ${ }^{3}$, situación no homologable y práctica que no representa el estándar en nuestro medio en la actualidad, donde la mayoría de las autoridades recomiendan la realización de cultivo para aquellos niños y adolescentes que presenten odinofagia, eritema faríngeo y ausencia de síntomas compatibles con un cuadro de vías aéreas superiores (fundamentalmente rinorrea y tos, pero también diarrea o rash cutáneo) $)^{4,5}$.

El puntaje FeverPAIN (disponible mediante una calculadora online en https://ctu1.phc.ox.ac.uk/feverpain/index.php) fue construido y utilizado en este estudio dado que mostró, en una cohorte ad hoc, mejores características operativas (mayor valor del área bajo la curva) que el tradicional puntaje de Centor. Vale la pena preguntarse si este puntaje resulta igualmente fiable en otras poblaciones.

Resulta también pertinente señalar que el presente trabajo no reportó la aparición de complicaciones asociadas a las distintas estrategias. Sin embargo, una publicación posterior, ${ }^{6}$ dejó en evidencia que si bien ciertos síntomas de manera aislada (otalgia e inflamación amigdalina severas) se correlacionan con la aparición de complicaciones supurativas, en la mayoría de los casos de complicaciones $(70 \%)$ estos síntomas no estuvieron presentes. Algo similar ocurrió con el puntaje FeverPAIN, donde se observaron complicaciones en un $73 \%$ de pacientes que puntuaron $\leq 2$.

\section{Conclusión del comentador}

La utilización de puntajes clínicos para decidir el tratamiento antimicrobiano de la faringitis aguda constituye una estrategia válida para el manejo de los adultos en cuanto a alivio sintomático más temprano y uso racional de antibióticos. En niños y adolescentes en quienes no se sospeche infección viral la determinación de infección estreptocócica continúa siendo el estándar de manejo en nuestro medio.

Gabriel Villalón [Servicio Medicina Familiar del Hospital Italiano de Buenos Aires. gabriel.villalon@ hospitalitaliano.org.ar]

Villalon G. La utilización de puntajes clínicos para decidir el tratamiento antimicrobiano de la faringitis aguda favorece el alivio sintomático y reduce el uso de antibióticos. Evid Act Pract Amb. 2015;18(2):40-41. Abr-Jun. Comentado de: Little P, Hobbs FD, Moore M, y col. Clinical score and rapid antigen detection test to guide antibiotic use for sore throats: randomised controlled trial of PRISM (primary care streptococcal management). BMJ 2013;347:f5806. PMID: 24114306.

\section{Referencias}

1. Chow AW, Doron S. Evaluation of acute pharyngitis in adults. In: UpToDate, Post TW (Ed), UpToDate, Waltham, MA. (último acceso marzo 2015).

2. NICE guideline development group. Prescribing of antibiotics for self-limiting respiratory tract infections in adults and children in primary care. www nice org uk/Guidance/CG692008.

3. Sharland M, Kendall H, Yeates D y col. Antibiotic prescribing in general practice and hospital admissions for peritonsillar abscess, mastoiditis, and rheumatic fever in children: time trend analysis. BMJ. 2005 Aug 6;331(7512):328-9. PMID: 15967760.

4. Patología aguda de la vía aérea superior. En: PROFAM, Salud del Anciano. Rubinstein E, et al Editores. 3ra edición. Buenos Aires: Fundación MF "Para el desarrollo de la Medicina Familiar y la Atención primaria de la salud", 2006

5. Wald E. Approach to diagnosis of acute infectious pharyngitis in children and adolescents. In: UpToDate, Post TW (Ed), UpToDate, Waltham, MA. (último acceso marzo 2015).

6. Little P y col. Predictors of suppurative complications for acute sore throat in primary care: prospective clinical cohort study. BMJ. 2013 Nov 25;347:f6867. PMID: 24277339. 